\title{
Coprinellus radians
}

National Cancer Institute

\section{Source}

National Cancer Institute. Coprinellus radians. NCI Thesaurus. Code C127699.

A species of saprobic mushroom-forming fungi in the family Psathyrellaceae. 